\title{
Toward low energy spread in plasma accelerators in quasilinear regime
}

\author{
Xiangkun Li, ${ }^{*}$ Phu Anh Phi Nghiem, and Alban Mosnier \\ CEA Saclay, 91191 Gif-Sur-Yvette, France
}

(Received 26 June 2018; published 8 November 2018)

\begin{abstract}
In this paper, we address the energy spread and slice energy spread of an externally injected electron beam in plasma wakefield accelerators operating in the linear or quasilinear regime. The energy spread is first derived taking into account the phase dependence of the wakefield along the finite-length bunch together with the dephasing during acceleration and found to be strongly dependent on the bunch length. This could be compensated by the beam loading effect, the energy spread from which is then derived and found to be nearly independent of the bunch length. However, the transverse dependence of the beam loading effect also makes the particles at the same longitudinal position experience different accelerating fields, introducing a significant slice energy spread. To estimate the slice energy spread, a theoretical analysis was conducted by taking the transverse betatron motion into account. As a study case, 3D simulations for the $5 \mathrm{GeV}$ laser-plasma acceleration stage of the European Plasma Research Accelerator with eXcellence in Applications project have been performed. Careful optimization of the parameters allows one to obtain an energy spread of $\leq 1 \%$ and a slice energy spread of $\leq 0.1 \%$, with good agreement between theories and simulations.
\end{abstract}

DOI: 10.1103/PhysRevAccelBeams.21.111301

\section{INTRODUCTION}

Plasma-based accelerators [1-4] have been considered as promising candidates to drive compact $\mathrm{x}$-ray light sources [5] or future lepton colliders [6] thanks to their ability to provide extremely high accelerating fields. The principle of such accelerators is to use a nonflat laser pulse or an electron beam to push the plasma electrons to the sides of its pathway by the ponderomotive force or the space charge force. As a result, a kind of cavity is formed in its wake, characterized by an area of electron deficiency surrounded by a border of electron excess. The resulting Coulomb force layout will make an electron bunch properly injected into the cavity on the axis experience a longitudinal accelerating field $E_{z}$ toward the front of the cavity and at the same time a transverse focusing field $E_{r}$ toward the center of the cavity. As the cavity is tiny, in the sub-mm range, the induced wakefields can be huge. Typically, an intense laser pulse or particle beam propagating in an ionized plasma could induce a plasma wave with electric fields in excess of the cold nonrelativistic wave breaking field, $E_{0}=m_{\mathrm{e}} c \omega_{\mathrm{p}} / e$ or $E_{0}(\mathrm{~V} / \mathrm{m}) \simeq 96 \sqrt{n_{\mathrm{p}}\left(\mathrm{cm}^{-3}\right)}$, where $m_{\mathrm{e}}$ and $e$ are the electron rest mass and charge, respectively, $c$ the speed of

\footnotetext{
*lixiangkun05@gmail.com
}

Published by the American Physical Society under the terms of the Creative Commons Attribution 4.0 International license. Further distribution of this work must maintain attribution to the author(s) and the published article's title, journal citation, and DOI. light in vacuum, $n_{\mathrm{p}}$ the plasma density, $\omega_{\mathrm{p}}=\sqrt{n_{\mathrm{p}} e^{2} / \epsilon_{0} m_{e}}$ the plasma wave frequency, and $\epsilon_{0}$ the electric constant. The wakefield could be up to $10^{3}$ times stronger than the field available in conventional if accelerators, but its longitudinal and transverse profiles are governed by the complex physical mechanisms which are difficult to handle [7], resulting in accelerated beams with a significant final energy spread $\left(\sigma_{E} / E\right)$ and/or slice energy spread $\left(\sigma_{E_{s}} / E\right)$, and making them not appropriate for most applications. Here $\sigma_{E}$ and $\sigma_{E_{s}}$ are the rms energy spreads for the total beam and for a beam slice, respectively, and $E$ is the average beam energy either for the total beam or for the slice.

The EuPRAXIA project [8] for example aims at providing beams at the final energy of $5 \mathrm{GeV}$, with $\sigma_{E} / E \leq 1 \%$ and $\sigma_{E_{s}} / E \leq 0.1 \%$, capable of driving an x-ray free electron laser. Reaching such a low energy spread requires careful optimization of the driver (laser or particle beam), the plasma and the input beam parameters, which implies many simulations, each being very time consuming. In order to limit the number of numerical simulations, we propose to investigate the linear theory describing the plasma wakefield in order to identify the parameters affecting the energy spread and to estimate their effects.

In the following sections, we start with the linear theory to derive, for a bi-Gaussian beam, the energy spread induced by the wakefield due to the fact that electrons at different longitudinal positions see different accelerating phases, which furthermore can progressively change along the acceleration process. Then we derive the effect of beam loading induced by the accelerated beam itself which can 
compensate the former [9-11]. However, the latter has also a strong radial dependence, which means particles within the same longitudinal slice experience nevertheless different accelerating fields. The slice energy spread so induced is derived, taking into account the betatron motion. Finally, we search to optimize the acceleration parameters accordingly in order to minimize $\sigma_{E} / E$ and $\sigma_{E_{s}} / E$ through simulations of the EuPRAXIA electron beam $(30 \mathrm{pC})$ externally injected by a plasma or rf injector.

\section{LINEAR THEORY}

In order to provide high energy gains in the $\mathrm{GeV}$ range, a strong enough wakefield is needed, making the choice of at least a quasilinear regime favored for the plasma wave. In such a regime, the plasma wakefield will no more be sinusoidal, as it steepens and its period lengthens [3]. However, we will still investigate the linear regime which is enough for identifying the parameters influencing the energy spread and for estimating their effects.

\section{A. Energy spread induced by plasma wakefield}

In the linear regime, the wakefield presents a sinusoidal form, i.e., $E_{z}(z)=E_{z 0} \cos \left[k_{\mathrm{p}}\left(z-v_{\mathrm{ph}} t\right)+\phi_{0}\right]=E_{z 0} \cos \phi$, where $E_{z 0}$ and $v_{\mathrm{ph}}=\beta_{\mathrm{ph}} c$ are the amplitude and phase velocity of the wakefield, respectively, $k_{\mathrm{p}}=\omega_{\mathrm{p}} / c$ the plasma wave number, $z \approx c t$ the position of the reference particle in the relativistic electron bunch in the laboratory frame, $\phi=$ $k_{\mathrm{p}}\left(z-v_{\mathrm{ph}} t\right)+\phi_{0}$ the relative phase of the particle to the crest of the wakefield, and $\phi_{0}$ the initial phase after injection.

The phase of an arbitrary particle in the electron bunch can be written into $\phi_{i}=\phi_{\mathrm{r}}+\Delta \phi_{i}$, where $\phi_{\mathrm{r}}$ and $\Delta \phi_{i}$ are the phase of the reference particle and the relative phase of the $i$ th particle to the reference one, respectively. Using Taylor series, we have to the second order

$$
\cos \phi_{i}=\cos \phi_{\mathrm{r}}-\Delta \phi_{i} \sin \phi_{\mathrm{r}}-\frac{\Delta \phi_{i}^{2}}{2} \cos \phi_{\mathrm{r}},
$$

when $\Delta \phi_{i} \ll 1$.

For an electron bunch driver, $v_{\mathrm{ph}}=c$ and the accelerating field seen by the reference particle is the same during the acceleration, $E_{z}(z)=E_{\mathrm{z} 0} \cos \phi_{0}$. The energy gain of the reference particle and the gain difference from an arbitrary particle are

$$
\begin{gathered}
W_{\text {ref }}=W_{\mathrm{m}} \cos \phi_{0}, \\
\Delta W_{i}=W_{i}-W_{\text {ref }}=W_{\mathrm{m}}\left[-\Delta \phi_{i} \sin \phi_{0}-\frac{\Delta \phi_{i}^{2}}{2} \cos \phi_{0}\right],
\end{gathered}
$$

respectively, where $W_{\mathrm{m}}=e E_{\mathrm{z} 0} L_{\mathrm{acc}}$ is the energy gain if $\phi_{0}=0$ and $L_{\text {acc }}$ is the total accelerating length. For a beam with a Gaussian longitudinal profile, i.e., $\rho_{\|}(\Delta \phi)=$ $\frac{1}{\sqrt{2 \pi} \sigma_{\phi}} \exp \left(-\Delta \phi^{2} / 2 \sigma_{\phi}^{2}\right)$ and $\sigma_{\phi}=k_{\mathrm{p}} \sigma_{z}$ with $\sigma_{z}$ the bunch length, the rms energy spread is obtained by

$$
\begin{aligned}
\sigma_{E}^{2} & =\int_{-\infty}^{\infty}\left(\Delta W_{i}\right)^{2} \rho_{\|} d\left(\Delta \phi_{i}\right)-\left[\int_{-\infty}^{\infty} \Delta W_{i} \rho_{\|} d\left(\Delta \phi_{i}\right)\right]^{2} \\
& =W_{\mathrm{m}}^{2}\left(\sin ^{2} \phi_{0} \sigma_{\phi}^{2}+\frac{1}{2} \cos ^{2} \phi_{0} \sigma_{\phi}^{4}\right) .
\end{aligned}
$$

When the acceleration is near the wave crest $\left(\phi_{0} \sim 0\right.$, $\sin \phi_{0} \sim 1$ and $\cos \phi_{0} \sim 1$ ), we have the relative energy spread:

$$
\sigma_{E} / E=\frac{\sqrt{2}}{2} k_{\mathrm{p}}^{2} \sigma_{z}^{2} .
$$

When the acceleration is off crest, we have

$$
\sigma_{E} / E=\tan \phi_{0} \cdot k_{\mathrm{p}} \sigma_{z}\left(1+\frac{1}{4} \cot ^{2} \phi_{0} k_{\mathrm{p}}^{2} \sigma_{z}^{2}\right) .
$$

Both equations show the dependence of the energy spread on the bunch length.

For a laser pulse driver, $v_{\text {ph }}<c$, the wakefields seen by the particles are continuously shifting, which is known as the dephasing effect. The energy gain of the reference particle and the gain difference from an arbitrary particle are obtained by integrating

$$
\begin{gathered}
W_{\text {ref }}=\int_{z_{0}}^{z_{1}} e E_{z}(z) \mathrm{d} z=W_{\mathrm{m}}\left(\sin \phi_{1}-\sin \phi_{0}\right), \\
\Delta W_{i}=W_{i}-W_{\text {ref }}=W_{\mathrm{m}}\left[\Delta \phi_{i}\left(\cos \phi_{1}-\cos \phi_{0}\right)\right. \\
\left.-\frac{\Delta \phi_{i}^{2}}{2}\left(\sin \phi_{1}-\sin \phi_{0}\right)\right]
\end{gathered}
$$

where $\phi_{1}$ is the exit phase of the reference particle, $W_{\mathrm{m}}=$ $\frac{e E_{z, 0}}{k_{\mathrm{p}}\left(1-\beta_{\mathrm{ph}}\right)}=2 e E_{z, 0} L_{\mathrm{dp}} / \pi$ is the maximum energy gain, and $L_{\mathrm{dp}}$ is the dephasing length. Equation (8) is obtained by using the Taylor series again. The rms energy spread thus is

$\sigma_{E}^{2}=W_{\mathrm{m}}^{2}\left[\left(\cos \phi_{1}-\cos \phi_{0}\right)^{2} \sigma_{\phi}^{2}+\frac{1}{2}\left(\sin \phi_{1}-\sin \phi_{0}\right)^{2} \sigma_{\phi}^{4}\right]$.

To the first order, the relative energy spread is

$$
\sigma_{E} / E \simeq \frac{\left|\cos \phi_{1}-\cos \phi_{0}\right|}{\sin \phi_{1}-\sin \phi_{0}} k_{\mathrm{p}} \sigma_{z},
$$

which also shows a dependence of the energy spread on the bunch length.

The quantity $\left(\cos \phi_{0}-\cos \phi_{1}\right) /\left(\sin \phi_{1}-\sin \phi_{1}\right)$ is plotted as a function of the exit phase $\phi_{1}$ for $\phi_{0}=0$ in Fig. 1, showing that the energy spread is increasing along the acceleration. Taking $n_{\mathrm{p}}=1 \times 10^{-17} \mathrm{~cm}^{-3}$ as an example, if the beam is accelerated from $\phi_{0}=0$ to $\phi_{1}=90$ degrees, 


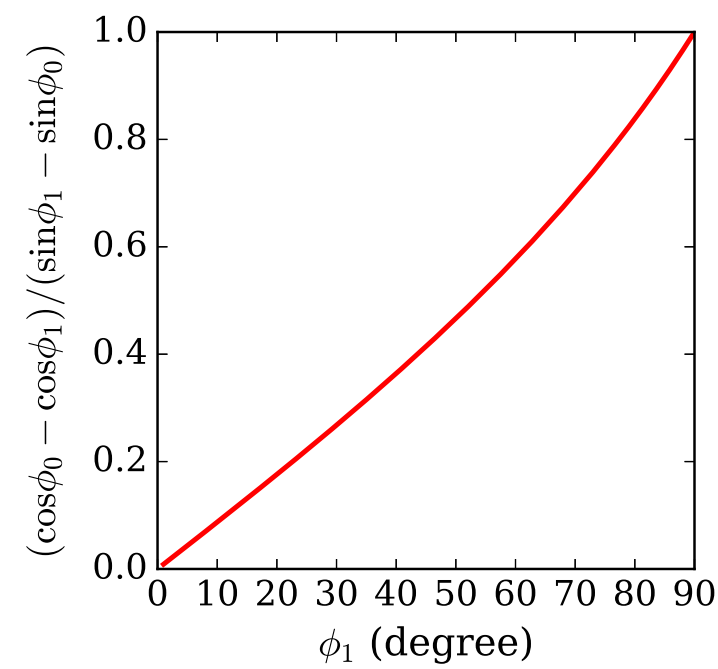

FIG. 1. The quantity $\left(\cos \phi_{0}-\cos \phi_{1}\right) /\left(\sin \phi_{1}-\sin \phi_{1}\right)$ as a function of the exit phase, showing how the relative energy spread evolves along with the acceleration.

the phase dependent contribution to the energy spread is unity and a final energy spread less than $1 \%$ means a very stringent bunch length: $k_{\mathrm{p}} \sigma_{z}<0.01$ or $\sigma_{z}<0.167 \mu \mathrm{m}$.

\section{B. Energy spread induced by beam loading effect}

The accelerated beam itself excites a comoving plasma wave when it goes through the plasma. The process that the wave produced by the accelerated beam modifies the wakefield in the plasma is referred to as beam loading. And its effect can no more be ignored when the bunch charge is high enough. In this paper, we use "plasma wakefield" to describe the wakefield induced by the driver and "beam loading effect" to describe that induced by the witness beam. In the linear or quasilinear regime, the beam loading effect can be calculated using perturbation theory [9]. For an arbitrary relativistic electron density of the form $n_{\mathrm{b}}(\xi, r)=n_{\|}(\xi) n_{\perp}(r)$, the longitudinal component of the wakefield can be written into

$E_{\mathrm{z}, \mathrm{b}}(\xi, r)=\frac{e}{\epsilon_{0}} \int_{-\infty}^{\xi} n_{\|}\left(\xi^{\prime}\right) \cos \left[k_{\mathrm{p}}\left(\xi-\xi^{\prime}\right)\right] d \xi^{\prime} \cdot R(r)$,

with

$$
R(r)=k_{\mathrm{p}}^{2} \int_{0}^{\infty} r^{\prime} \mathrm{d} r^{\prime} n_{\perp}\left(r^{\prime}\right) K_{0}\left(k_{\mathrm{p}}\left|\vec{r}-\overrightarrow{r^{\prime}}\right|\right),
$$

where "b" denotes beam related parameters, $K_{0}$ the zerothorder modified Bessel function, and $\xi$ and $r$ the longitudinal and radial coordinates in the comoving frame, respectively. Let us study a bi-Gaussian bunch profile, that is, $n_{\mathrm{b}}(\xi, r)=$ $n_{\mathrm{b}} \exp \left(-\xi^{2} / 2 \sigma_{z}^{2}\right) \exp \left(-r^{2} / 2 \sigma_{r}^{2}\right)$, with $n_{\mathrm{b}}=Q_{\mathrm{b}} /\left[e(2 \pi)^{1.5}\right.$ $\sigma_{z} \sigma_{r}^{2}$ ], $Q_{\mathrm{b}}$ the bunch charge, $\sigma_{r}$ and $\sigma_{z}$ the rms beam size and bunch length, respectively. For this profile, the on-axis longitudinal field at the bunch center $(\xi=0, r=0)$ is

$$
\begin{aligned}
E_{\mathrm{z}, \mathrm{b}}(0) & =\frac{e}{\epsilon_{0}} \int_{-\infty}^{0} n_{\|}\left(\xi^{\prime}\right) \cos \left[k_{\mathrm{p}}\left(\xi-\xi^{\prime}\right)\right] d \xi^{\prime} \cdot R(0) \\
& =\sqrt{\frac{\pi}{2}} \frac{e n_{\mathrm{b}}}{\epsilon_{0}} \sigma_{z} \exp \left(-\frac{k_{\mathrm{p}}^{2} \sigma_{z}^{2}}{2}\right) R(0),
\end{aligned}
$$

and the expression for $R(0)$ is [12]

$$
R(0)=\left(\frac{k_{\mathrm{p}}^{2} \sigma_{r}^{2}}{2}\right) \exp \left(-\frac{k_{\mathrm{p}}^{2} \sigma_{r}^{2}}{2}\right) \Gamma\left(0, \frac{k_{\mathrm{p}}^{2} \sigma_{r}^{2}}{2}\right)
$$

where $\Gamma$ is the incomplete gamma function. In the limit of $k_{\mathrm{p}} \sigma_{r} \ll 1, R(0)$ can be expanded asymptotically as

$$
R(0)=k_{\mathrm{p}}^{2} \sigma_{r}^{2}\left[0.058-\ln \left(k_{\mathrm{p}} \sigma_{r}\right)\right] .
$$

Insertion of $n_{\mathrm{b}}$ and $R(0)$ into Eq. (13) leads to

$$
\begin{aligned}
E_{\mathrm{z}, \mathrm{b}}(0) & =\frac{Q_{\mathrm{b}}}{4 \pi \epsilon_{0}} k_{\mathrm{p}}^{2} e^{-\frac{k_{\mathrm{p}}^{2} \sigma_{\mathrm{z}}^{2}}{2}}\left[0.058-\ln \left(k_{\mathrm{p}} \sigma_{r}\right)\right] \\
& \propto Q_{\mathrm{b}} n_{\mathrm{p}}\left[0.058-\ln \left(k_{\mathrm{p}} \sigma_{r}\right)\right] .
\end{aligned}
$$

The on-axis longitudinal field, to the first order of Taylor series, is

$$
E_{\mathrm{z}, \mathrm{b}}(\xi) \simeq E_{\mathrm{z}, \mathrm{b}}(0)+E_{\mathrm{z}, \mathrm{b}}^{\prime}(0) \xi,
$$

where $E_{\mathrm{z}, \mathrm{b}}^{\prime}(0)$ is the derivative of $E_{\mathrm{z}, \mathrm{b}}$ with respect to $\xi$ at $\xi=0$ and $r=0$ and can be obtained by applying the Leibniz's rule to Eq. (11),

$$
\begin{aligned}
E_{\mathrm{z}, \mathrm{b}}^{\prime}(0) & =\frac{e n_{\mathrm{b}}}{\epsilon_{0}}\left[1+\sqrt{\frac{\pi}{2}}\left(i k_{\mathrm{p}} \sigma_{z}\right) \operatorname{erf}\left(\frac{i k_{\mathrm{p}} \sigma_{z}}{\sqrt{2}}\right) e^{-\frac{k_{\mathrm{p}}^{2} \sigma_{z}^{2}}{2}}\right] R(0) \\
& \simeq \frac{e n_{\mathrm{b}}}{\epsilon_{0}}\left[1-k_{\mathrm{p}}^{2} \sigma_{z}^{2} e^{-\frac{k_{\mathrm{p}}^{2} \sigma_{z}^{2}}{2}}\right] R(0)
\end{aligned}
$$

where $\operatorname{erf}(x)=2 / \sqrt{\pi}\left(x-x^{3} / 3+\cdots\right)$ is used to replace the error function term, also in the limit of $k_{\mathrm{p}} \sigma_{z} \ll 1$.

Figure 2 shows the on-axis longitudinal fields calculated by integrating Eq. (11) and by the approximated solution Eq. (17), indicating good agreement for $-\sigma_{z}<\xi<\sigma_{z}$ where most of the particles reside.

Using this approximation, we obtain the rms energy spread induced on the beam per unit length:

$$
\frac{\sigma_{E}}{L} \simeq e E_{\mathrm{z}, \mathrm{b}}^{\prime}(0) \sigma_{z}=\frac{e Q_{\mathrm{b}} k_{\mathrm{p}}^{2}}{(2 \pi)^{\frac{3}{2}} \epsilon_{0}}\left(1-k_{\mathrm{p}}^{2} \sigma_{z}^{2}\right)\left[0.058-\ln \left(k_{\mathrm{p}} \sigma_{r}\right)\right] .
$$

While the energy spread induced by the plasma wakefield is directly dependent on the bunch length, the energy spread induced by the beam loading effect is not, in the limit of $k_{\mathrm{p}} \sigma_{z} \ll 1$. The latter is more sensitive to the beam size as the $\ln \left(k_{\mathrm{p}} \sigma_{r}\right)$ term implies. Therefore, for either a 


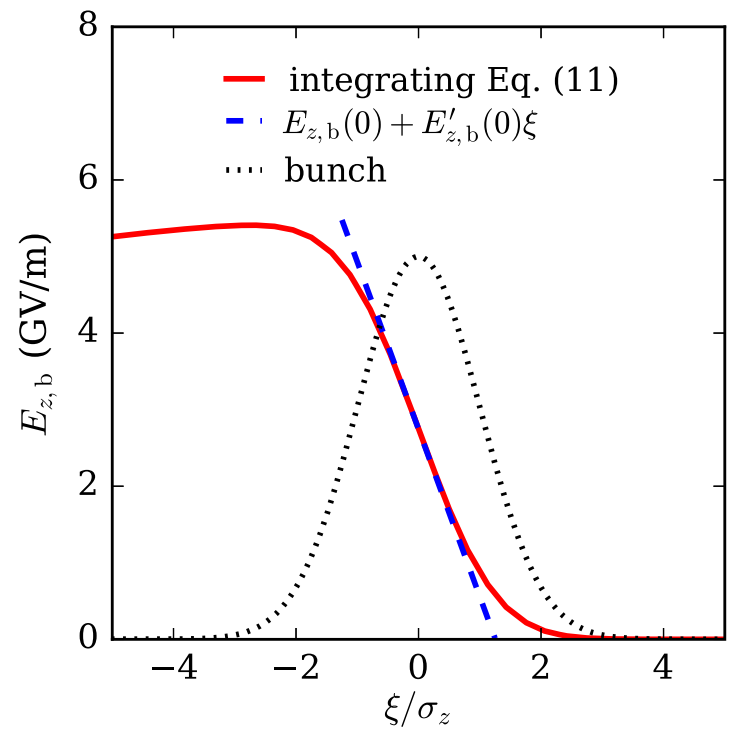

FIG. 2. On-axis longitudinal fields along the bunch (dotted black line) given by Eq. (11) (solid red line) and the approximation (17) (dashed blue line), respectively, with $n_{\mathrm{p}}=1 \times 10^{17} \mathrm{~cm}^{-3}$, $\sigma_{r}=\sigma_{z}=1 \mu \mathrm{m}$, and $Q_{\mathrm{b}}=30 \mathrm{pC}$.

particle beam driver or a laser pulse driver, we can choose a proper bunch length such that the two effects cancel out as much as possible.

\section{Slice energy spread induced by transverse dependence of the fields}

In deriving the analytical expressions for the energy spread, only the on-axis longitudinal fields are considered. However, both the plasma wakefield and beam loading effect have a transverse dependence, which means particles within the same longitudinal slice, i.e., same $\xi$, will see different accelerating fields depending on their radial positions. The accelerating field experienced by one particle is the addition of the wakefields, that is

$$
E_{\text {acc }}(\xi, r, z)=E_{z}(r, z+\xi)+E_{z, \mathrm{~b}}(\xi, r, z) .
$$

Note here $E_{\mathrm{z}, \mathrm{b}}$ is written as a function of $z$ in case that the plasma density seen by the beam evolves gradually due to dephasing. The first term on the right-hand side of Eq. (20) representing the plasma wakefield has the form of $E_{z} \sim \exp \left(-2 r^{2} / r_{0}^{2}\right)$, where $r_{0}$ is the driver's size. It is $r$ independent near the axis when the beam size is much smaller than the driver's size, i.e., $\sigma_{r} \ll r_{0}$ [3], which is often the case. The second term on the right-hand side representing the beam loading effect can be written into

$$
E_{\mathrm{z}, \mathrm{b}}(\xi, r, z)=E_{\mathrm{z}, \mathrm{b}}(\xi, z) \hat{R}(r),
$$

so as to point out its significant transverse dependence, where $\hat{R}(r)=R(r) / R(0)$. The distributions of $\hat{R}(r)$ numerically solved from Eq. (12) are shown in Fig. 3

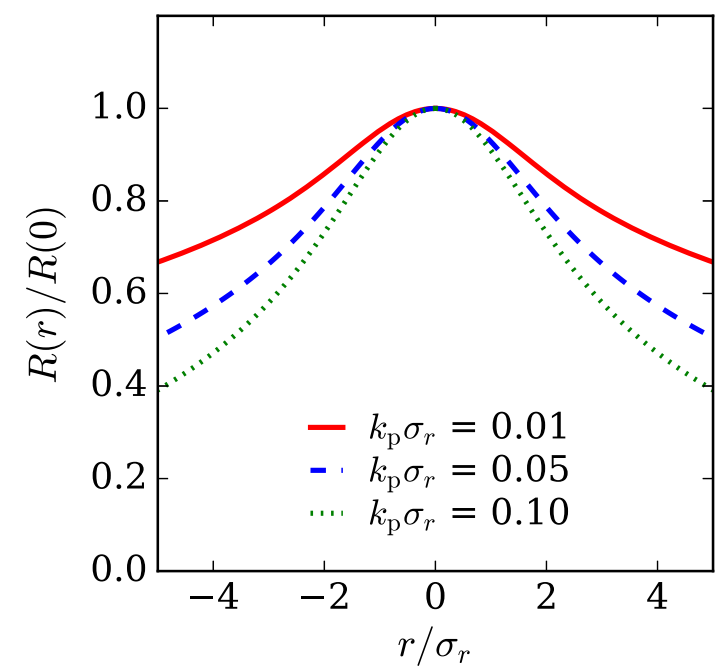

FIG. 3. Transverse dependence of the beam loading effect, with $n_{\mathrm{p}}=1 \times 10^{17} \mathrm{~cm}^{-3}$.

for various beam sizes with the plasma density $n_{\mathrm{p}}=$ $1.0 \times 10^{17} \mathrm{~cm}^{-3}$. As $E_{\mathrm{z}, \mathrm{b}}$ is positive inside the electron beam, Fig. 3 implies that the off-axis electrons are less decelerated than those on the axis.

Because of the betatron motion induced by the wakefield focusing gradient, the transverse coordinate of one particle is changing fast with time, as well as the decelerating field it experiences. To derive the slice energy spread, the betatron motion must be taken into account. Note that only the wake field gradient should be considered because that of the beam loading is negligible for a relatively low bunch charge, as demonstrated in [13] (when the bunch charge goes very high, the beam loading effect would dominate and cancel the accelerating field, making the bubble regime more adequate [10], which can load much higher bunch charge). For the single particle differential equation $x^{\prime \prime}+K x=0$, its solution reads [14]

$$
x=x_{0} \cos \varphi_{x}+\frac{x_{0}^{\prime}}{\sqrt{K}} \sin \varphi_{x},
$$

where $K$ is the focusing strength, $\varphi_{x}=\sqrt{K} z$ is the phase advance. When the beam is matched, we have $\alpha_{\mathrm{m}}=0$ and $\beta_{\mathrm{m}}=1 / \sqrt{K}$, where $\alpha_{\mathrm{m}}$ and $\beta_{\mathrm{m}}$ stand for the matched Twiss parameters. If there is no acceleration, the particle has a harmonic motion and its trajectory in the normalized trace space $\left(x / \sqrt{\beta}, \sqrt{\beta} x^{\prime}\right)$ would be a closed circle, as shown in Fig. 4. But with acceleration, the oscillation amplitude is gradually decreasing and the trajectory becomes a spiral toward the origin. To estimate the effect of acceleration on the betatron motion, consider the dependence of $\beta$ on the beam size $\sigma_{x}$, the normalized beam emittance $\varepsilon_{n, x}$ and the energy relativistic factor $\gamma_{\mathrm{r}}: \beta=\sigma_{x}^{2} /\left(\varepsilon_{n, x} / \gamma_{\mathrm{b}}\right)$, which leads to $K=\left(\varepsilon_{n, x} / \gamma_{\mathrm{r}} \sigma_{x}^{2}\right)^{2}$ when matched. The oscillation period is related to $K$ by $c T=2 \pi / \sqrt{K}$, and the relative energy gain during the time $T$ is 


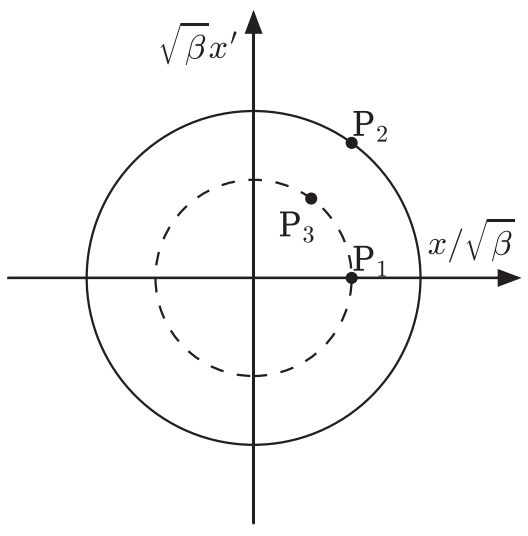

FIG. 4. Illustration of particles' motions in 2D trace space.

$$
\frac{\Delta E}{E}=\frac{e E_{\mathrm{acc}} \cdot c T}{\gamma_{\mathrm{r}} m_{e} c^{2}}=\frac{e E_{\mathrm{acc}}}{m_{e} c^{2}} \frac{2 \pi \sigma_{x}^{2}}{\varepsilon_{n, x}} .
$$

Taking typical beam parameters, $\sigma_{x} \sim 0.5 \mu \mathrm{m}, \varepsilon_{n, x} \sim$ $1 \mathrm{~mm} \mathrm{mrad}$, and $E_{\mathrm{acc}} \sim 10 \mathrm{GV} / \mathrm{m}$, we get $\Delta E / E \approx 0.03$. The small change in beam energy implies that even with acceleration, the particle's trajectory during the time $T$ is still close to a circle thanks to the very fast oscillation.

Since the focusing field in the plasma wave is cylindrically symmetric, the motion in $\left(y, y^{\prime}\right)$ trace space is similar,

$$
y=y_{0} \cos \varphi_{y}+\frac{y_{0}^{\prime}}{\sqrt{K}} \sin \varphi_{y} .
$$

Together, the radial coordinate of the particle can be written into

$$
r^{2}=A_{x}^{2} \cos ^{2} \varphi_{0}+A_{y}^{2} \cos ^{2}\left(\varphi_{0}+\Delta \varphi\right),
$$

where $A_{x}=\sqrt{x_{0}^{2}+x_{0}^{\prime 2} / K}$ and $A_{y}=\sqrt{y_{0}^{2}+y_{0}^{\prime 2} / K}$ are the oscillation amplitudes in $x$ and $y$ planes, respectively, $\varphi_{0}$ the initial phase in $\left(x, x^{\prime}\right)$ trace space, and $\Delta \varphi$ the relative phase between $\left(x, x^{\prime}\right)$ and $\left(y, y^{\prime}\right)$ trace spaces.

Within a slice, all the particles experience the same focusing strength and therefore have the same betatron period. To obtain the slice energy spread, it is necessary to average their energy gains within one or multiple turns of oscillation. To illustrate this, consider the motion of particles in Fig. 4. The energy gains of $P_{1}$ and $P_{3}$ will be the same after one turn because of their same trajectories. The energy gains of $P_{1}$ and $P_{2}$ will be very different after one turn because of their different trajectories, although they have the same transverse coordinates at the beginning of oscillations.

For a slice located at $\xi$, the difference in accelerating field between a particle oscillating around the axis and a particle fixed on the axis is $e E_{\mathrm{z}, \mathrm{b}}(\xi, z)[1-\hat{R}(r)]$. And the difference in energy gain during the period $T$ or the distance $L=c T$ is

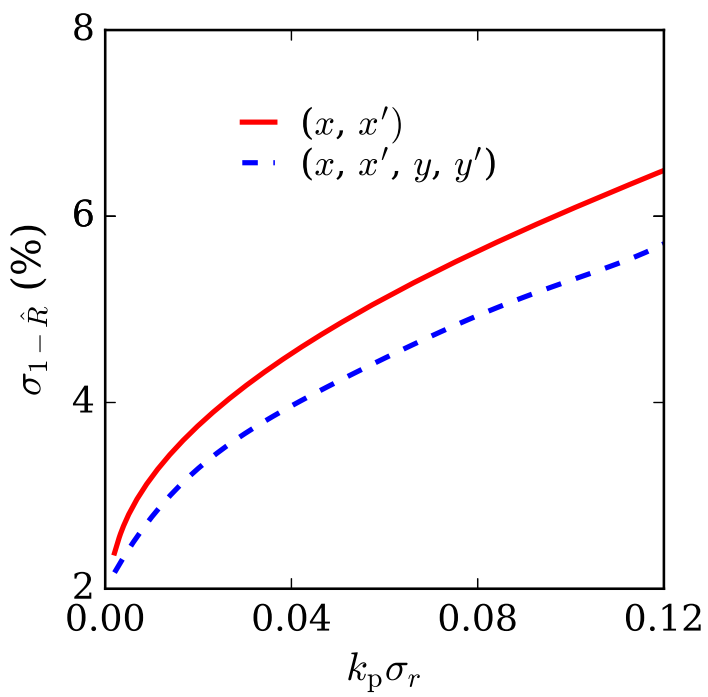

FIG. 5. $\sigma_{1-\hat{R}}$ as a function of the normalized beam size numerically solved in 2D (solid red line) and 4D (dashed blue line) trace space, respectively.

$$
\Delta W_{s}=e E_{\mathrm{z}, \mathrm{b}}(\xi, z) L \cdot\left\langle 1-\hat{R}\left(A_{x}, A_{y}, \varphi_{0}, \Delta \varphi\right)\right\rangle .
$$

To solve the rms energy spread, the distributions of $A_{x}, A_{y}$, $\varphi_{0}$, and $\Delta \varphi$ should be known first. As demonstrated in Eq. (A6), $f\left(A_{x}\right)$ [or $f\left(A_{y}\right)$ ] follows exactly the same distribution as $n_{\perp}$. Here we use $f$ to denote the probability distribution function (PDF). Since the particles are randomly distributed in the trace space, we have $f\left(\varphi_{0}\right)=$ $f(\Delta \varphi)=1 / 2 \pi$. With these, the $\hat{R}$ related contribution to the rms is obtained by

$$
\begin{aligned}
\left\langle[1-\hat{R}]^{2}\right\rangle= & \int_{-\pi}^{\pi} \int_{-\pi}^{\pi} \int_{0}^{\infty} \int_{0}^{\infty}[1-\hat{R}]^{2} f\left(A_{x}\right) f\left(A_{y}\right) f(\Delta \varphi) \\
& \times f\left(\varphi_{0}\right) d A_{x} d A_{y} d(\Delta \varphi) d \varphi_{0} \\
\langle 1-\hat{R}\rangle= & \int_{-\pi}^{\pi} \int_{-\pi}^{\pi} \int_{0}^{\infty} \int_{0}^{\infty}[1-\hat{R}] f\left(A_{x}\right) f\left(A_{y}\right) f(\Delta \varphi) \\
& \times f\left(\varphi_{0}\right) d A_{x} d A_{y} d(\Delta \varphi) d \varphi_{0} \\
\sigma_{1-\hat{R}}= & \sqrt{\left\langle[1-\hat{R}]^{2}\right\rangle-\langle 1-\hat{R}\rangle^{2}}
\end{aligned}
$$

And the rms slice energy spread is given by

$$
\sigma_{E_{s}}=e E_{\mathrm{z}, \mathrm{b}}(\xi, z) L \cdot \sigma_{1-\hat{R}}
$$

The multiple integrals in Eq. (27) can be solved efficiently using the Monte Carlo method, knowing the distributions of $A_{x}, A_{y}, \varphi_{0}$, and $\Delta \varphi$. The numerical results are shown in Fig. 5, with $\sigma_{1-\hat{R}}$ also being solved in 2D trace space. It can be found that the smaller the normalized beam size, the less the transverse dependence will contribute to the slice energy spread. As the beam is accelerated, the beam size is reduced and the effect of the transverse dependence on 


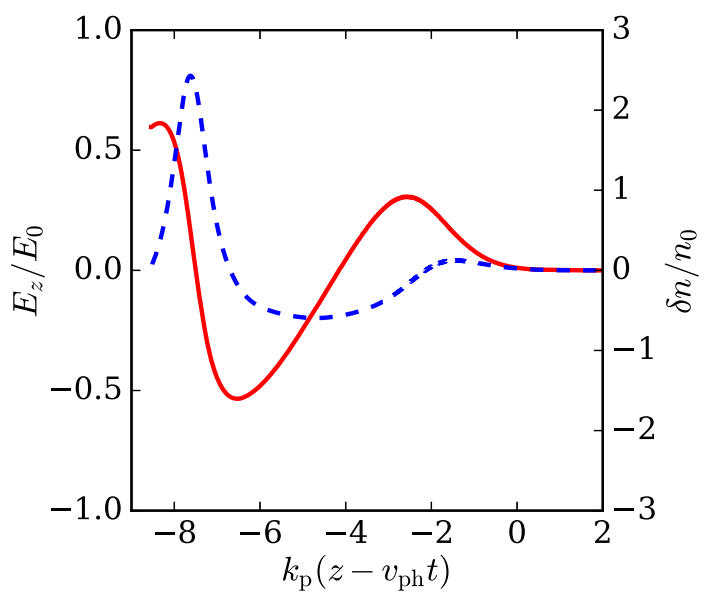

FIG. 6. On-axis longitudinal electric field (solid red line) and plasma density (dashed blue line), with $n_{\mathrm{p}}=1.5 \times 10^{17} \mathrm{~cm}^{-3}$ and $a_{0}=\sqrt{2}$.

the slice energy spread is weakened. Moreover, $\sigma_{1-\hat{R}}$ is found to be higher in $2 \mathrm{D}$ trace space than in $4 \mathrm{D}$ trace space, implying that 2D simulations in a slab geometry would lead to an overestimated slice energy spread.

Furthermore, assume that the beam size and the plasma density seen by the accelerated beam do not change significantly during the whole acceleration process. The former holds when the beam energy is very high and the latter is valid for a particle beam driver. For a laser pulse driver operating in the quasilinear regime, the plasma density seen by the beam does not change too much, either, as illustrated later in Fig. 6. These assumptions mean that the beam loading effect [determined by $n_{\mathrm{p}}$ and $k_{\mathrm{p}} \sigma_{r}$ as in Eq. (16)] will be constant despite the acceleration, or $E_{\mathrm{z}, \mathrm{b}}(\xi, z)=E_{\mathrm{z}, \mathrm{b}}(\xi)$, and its contribution to the slice energy spread will be the same period after period. As a result, the final slice energy spread will be

$$
\sigma_{E_{s}}=e E_{\mathrm{z}, \mathrm{b}}(\xi) L_{\mathrm{acc}} \cdot \sigma_{1-\hat{R}}
$$

and the relative slice energy spread will be

$$
\sigma_{E_{s}} / E \simeq \frac{e E_{\mathrm{z}, \mathrm{b}}(\xi) L_{\mathrm{acc}} \cdot \sigma_{1-\hat{R}}}{W_{\mathrm{ref}}}=\frac{E_{\mathrm{z}, \mathrm{b}}(\xi)}{\left\langle E_{\mathrm{acc}}\right\rangle} \cdot \sigma_{1-\hat{R}}
$$

where $\left\langle E_{\text {acc }}\right\rangle \simeq \Delta W_{\text {ref }} / L_{\text {acc }}$ is the accelerating field averaged on the whole accelerating length.

\section{Comparison with nonlinear regime}

The beam loading effect in linear regime and strong nonlinear or bubble regime has been studied previously [9-11]. A triangular bunch shape has been found to be able to produce a completely flat accelerating field along the beam axis in both regimes, thus eliminating the energy spread. In the bubble regime, as the witness beam sees no background plasma electrons inside the bubble, the field it induces is much weaker than that in the linear regime. As a result, it requires a much higher bunch charge (e.g., $100 \mathrm{pC}-\mathrm{nC}$ ) to generate the ideally constant field. When the bunch charge is not high enough, a much shorter bunch length would be required to mitigate the phase-dependent effect of the accelerating field following Eq. (6) or (9). For a moderate bunch charge (e.g., $30 \mathrm{pC}$ in the EuPRAXIA project) the quasilinear regime is more favorable because the beam loading effect is strong enough to cancel with the accelerating field, in the presence of a relatively long bunch length. Therefore, in term of energy spread it depends on the bunch charge to be accelerated and the bunch length available whether to choose the quasilinear regime or the bubble regime. In term of slice energy spread the bubble regime prevails over the quasilinear regime, since it is free of radial dependence [10,11].

\section{SIMULATIONS AND ENERGY SPREAD MINIMIZATION}

\section{A. Simulation configuration}

Though the expressions established above describe the laws governing the energy spread in the context of linear regime, it is worth examining them with numerical simulations in the more efficient quasilinear regime to see how they can help minimize the energy spread.

We consider one of the EuPRAXIA laser wakefield acceleration (LWFA) configurations [8], comprising a powerful laser driver to induce a strong wakefield capable of accelerating a $30 \mathrm{pC}$ electron beam externally injected at $150 \mathrm{MeV}$ to $5 \mathrm{GeV}$. One of the challenging requirement is that the final energy spread will not exceed $1 \%$ and $0.1 \%$ in terms of $\sigma_{E} / E$ and $\sigma_{E_{s}} / E$, respectively. The main parameters used in the simulations are listed in Table I. The beam has a Gaussian profile both transversely and longitudinally (therefore no bunch shaping). The normalized vector potential of

TABLE I. Simulation parameters for the LWFA.

\begin{tabular}{lcc}
\hline \hline Variable & Value & Unit \\
\hline Laser & & \\
$\quad$ Strength $a_{0}$ & $1-2$ & \\
Spot size $k_{\mathrm{p}} r_{\mathrm{L}}$ & $\sim 3$ & \\
$\quad$ Duration $k_{\mathrm{p}} \sigma_{\mathrm{L}}$ & $\sqrt{2}$ & \\
Plasma & & \\
$\quad$ Density $n_{\mathrm{p}}$ & $1-2$ & $10^{17} \mathrm{~cm}^{-3}$ \\
Accelerating length $L_{\text {acc }}$ & $\sim 30$ & $\mathrm{~cm}$ \\
$\quad$ Channel depth $\Delta n / \Delta n_{\mathrm{c}}$ & $<1$ & \\
Electron & & \\
Charge $Q$ & 30 & $\mathrm{pC}$ \\
Energy $E_{k}$ & 150 & $\mathrm{MeV}$ \\
Energy spread $\sigma_{E} / E$ & 0.5 & $\%$ \\
Emittance $\varepsilon_{n, x}$ & 1.0 & $\pi \mathrm{mmmrad}$ \\
Beam size $\sigma_{x}$ & $\sim 1$ & $\mu \mathrm{m}$ \\
Bunch length $\sigma_{z}$ & $1-3$ & $\mu \mathrm{m}$ \\
\hline
\end{tabular}


the laser pulse is defined as $a^{2}(\xi, r)=a_{0}^{2} e^{-2 r^{2} / r_{\mathrm{L}}^{2}-\xi^{2} / \sigma_{\mathrm{L}}^{2}}$, where $r_{\mathrm{L}}$ is the spot size and $\sigma_{\mathrm{L}}$ the rms pulse length. The critical plasma channel is given by $\Delta n_{\mathrm{c}}=\left(\pi r_{e} r_{\mathrm{L}}^{2}\right)^{-1}$, where $r_{e}=e^{2}\left(4 \pi \epsilon_{0} m_{e} c^{2}\right)^{-1}$ is the classical electron radius.

The input beam parameters look rather challenging, seeing the high bunch charge and simultaneously the small energy spread, small beam size and small bunch length. Different teams in charge of achieving those beam qualities are however not far from reaching these objectives. Different techniques are under consideration, like resonant multipulse ionization injection [15], ionization injection [16], magnetically controlled injection [17,18], downramp injection [19], or conventional photoinjector [20]. The option of increasing the beam energy up to $350 \mathrm{MeV}$ for photoinjectors is also considered, in order to combat the space charge effects. For the bunch length, a bunch compressor should be available in the transfer line.

For the minimization of the energy spread and slice energy spread, we have performed simulations with different laser and plasma parameters. For each set of parameters, the plasma channel depth and the beam size have been optimized first [13]: the channel depth is chosen so that the laser propagates without neither significant overfocusing nor defocusing [21]; the initial beam size is matched to the transverse focusing force at the plasma entrance so that the emittance is preserved during the acceleration $[22,23]$. The simulations were carried out by the 3D Particle-In-Cell code WARP [24] in the boosted frame. The dimension of the moving window was typically $228 \times 228 \times 150 \mu \mathrm{m}$. The transverse grid size was $\lambda_{0} / 2$ to $\lambda_{0}$ and the longitudinal grid size was $\lambda / 32$ with $\lambda_{0}=$ $0.8 \mu \mathrm{m}$ the laser wavelength, corresponding to at least $285 \times$ $285 \times 5524$ cells. There was only one particle per grid for the plasma and about 200 particles per grid for the electron beam. The coarser transverse grid size has been compared to the finer one and the results agreed well. Therefore, the coarser one was typically used to save simulation time. The length of the moving window was longer when the plasma density was lower, ensuring that the first wave crest behind the laser pulse was well located inside the moving window. The boosted factor was $\gamma_{\text {boost }}=16$.

Figure 6 shows the typical longitudinal laser wakefield together with the plasma density distribution. In the quasilinear regime, the perturbation of plasma density is significant, leaving an almost constant density channel for the accelerated beam.

\section{B. Minimization of total energy spread}

The idea here is to minimize the energy spread by varying the bunch length at injection. According to Eq. (10), this will change the energy spread induced by the plasma wakefield such that it is the best balanced by that induced by the beam loading effect which is invariant as regards the bunch length [Eq. (19)].

Figure 7(a) shows the resulting energy spread along the acceleration path for 5 different bunch lengths with the
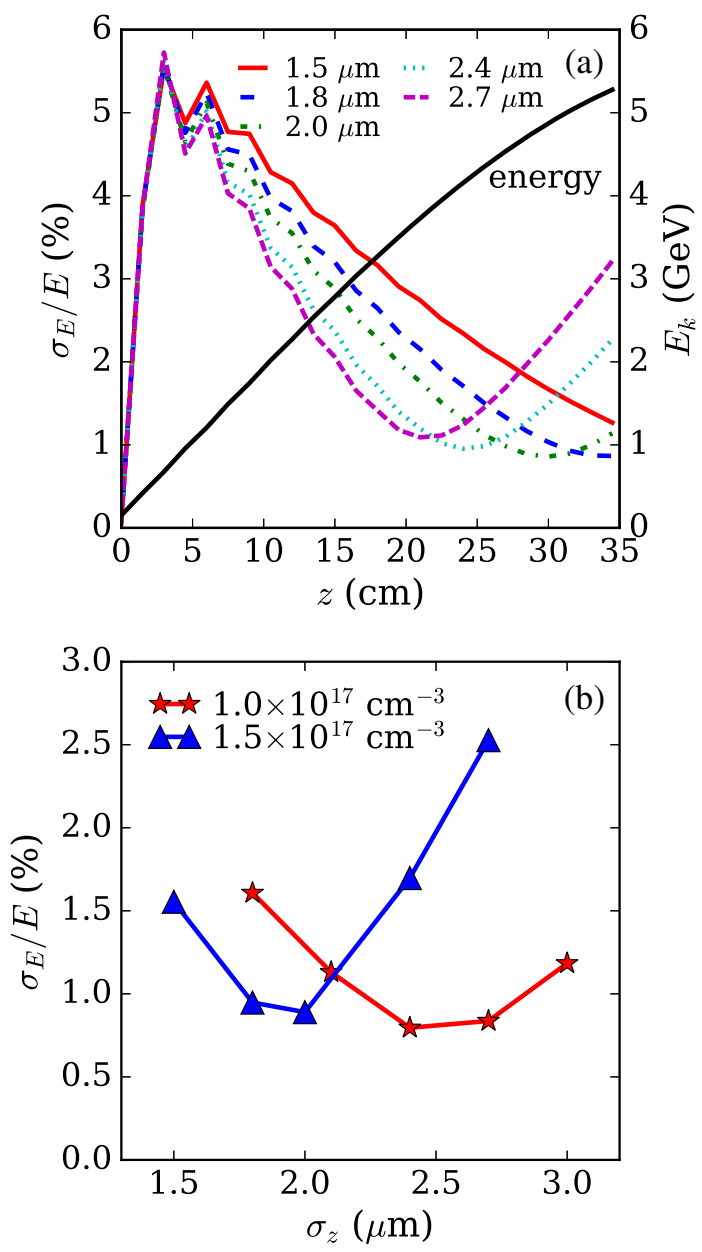

FIG. 7. (a) Evolutions of beam energy spread for the plasma density $n_{\mathrm{p}}=1.5 \times 10^{17} \mathrm{~cm}^{-3}$ and various bunch lengths and (b) energy spread vs the bunch length for $n_{\mathrm{p}}=1.0$ and $1.5 \times 10^{17} \mathrm{~cm}^{-3}$. The laser strength is $a_{0}=\sqrt{2}$.

plasma density $n_{\mathrm{p}}=1.5 \times 10^{17} \mathrm{~cm}^{-3}$ and the laser strength $a_{0}=\sqrt{2}$. At the plasma entrance, due to the only presence of the strong beam loading effect, there is an abrupt but same increase of energy spread for all the bunch lengths. Then as the acceleration continues the effect from the plasma wakefield plays its role more and more according to Eq. (10) or Fig. 1, i.e., depending on the bunch length and therefore compensating more or less with the unchanged beam loading effect.

The resulting energy spread is collected at the targeted energy of $5 \mathrm{GeV}$ in Fig. 7(b) for two different plasma densities $n_{\mathrm{p}}=1.0$ and $1.5 \times 10^{17} \mathrm{~cm}^{-3}$ and the laser strength $a_{0}=\sqrt{2}$. We can see that for each case, there exists a bunch length for which the energy spread is minimized and the minimum is around the requirement of $1 \%$. Those bunch lengths are just less than $3 \mu \mathrm{m}$ (see Table I) as imposed by Free-Electron-Laser requirements in the EuPRAXIA project. A bunch compressor is foreseen in the transfer line for achieving the desired bunch length. 

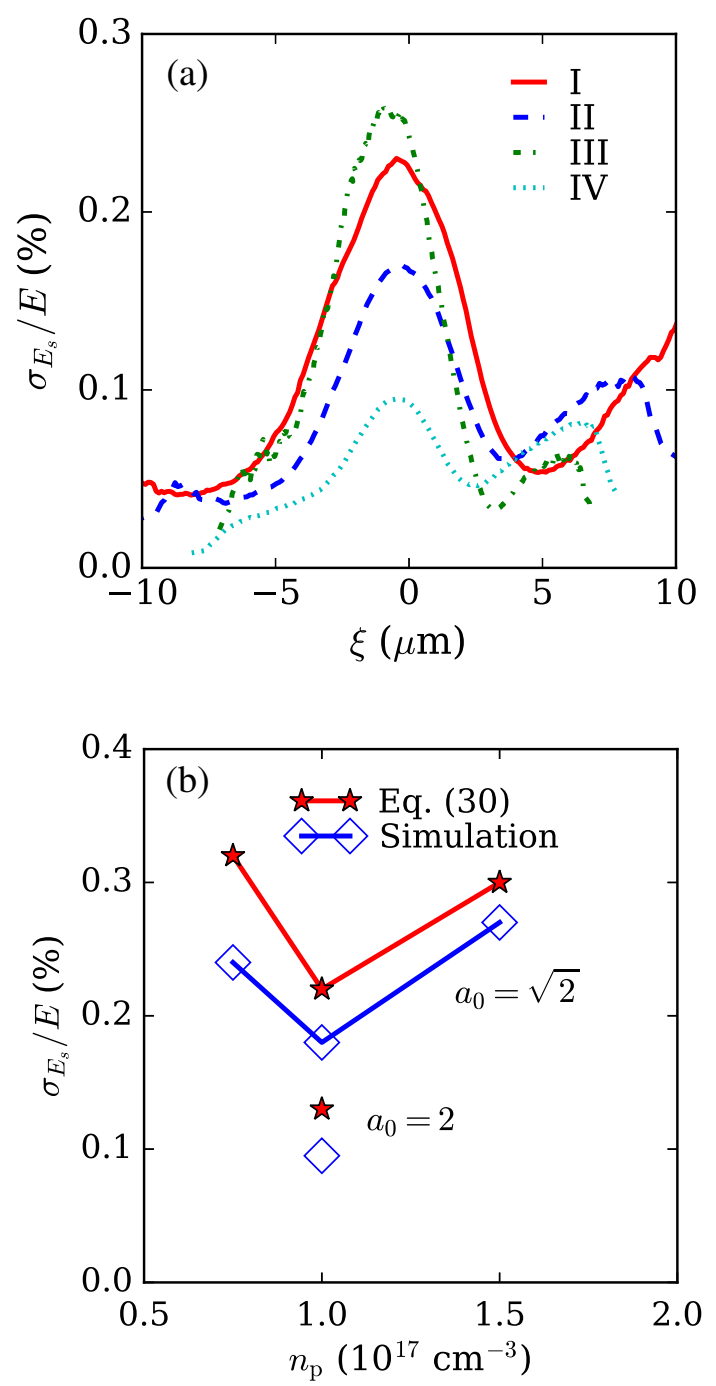

FIG. 8. (a) Slice energy spread distributions for $a_{0}=\sqrt{2}$ and $n_{\mathrm{p}}=0.75,1.0,1.5 \times 10^{17} \mathrm{~cm}^{-3}$ (I to III) and for $a_{0}=2$ and $n_{\mathrm{p}}=1.0 \times 10^{17} \mathrm{~cm}^{-3}$ (IV) and (b) slice energy spread at the bunch center $(\xi=0)$ as a function of the plasma density and laser strength.

\section{Optimization of slice energy spread}

According to Eq. (30), the slice energy spread is dependent on the acceleration term, $\left\langle E_{\text {acc }}\right\rangle$, and the beam loading term, $E_{\mathrm{z}, \mathrm{b}}$. On one hand, $\left\langle E_{\mathrm{acc}}\right\rangle$ is determined by the amplitude of the laser wakefield which increases with the plasma density and by the acceleration phase interval which tends to move toward the crest in a lower plasma density considering the dephasing effect. This implies there exists a best plasma density for maximizing $\left\langle E_{\text {acc }}\right\rangle$. On the other hand, $E_{\mathrm{z}, \mathrm{b}}$ is proportional to $\sqrt{n_{\mathrm{p}}}$. Therefore by tuning the plasma density, the slice energy spread could be reduced. Moreover, the higher the laser strength, the stronger the laser wakefield becomes and the lower the remnant plasma density is left for the witness beam, which in return means a lower beam loading effect, making the

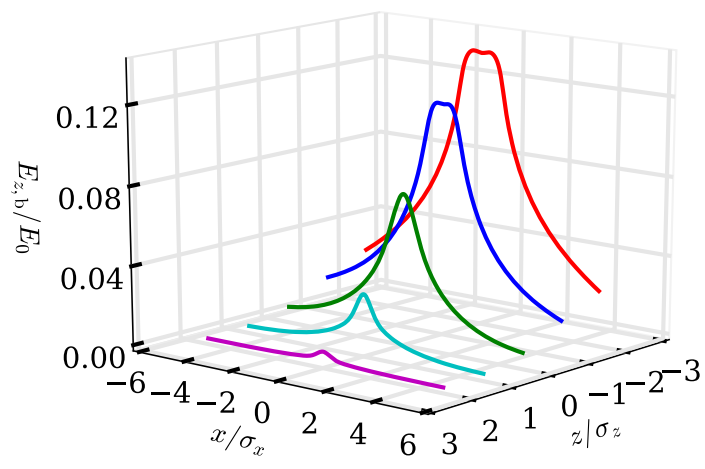

FIG. 9. 2D distributions of $E_{\mathrm{z}, \mathrm{b}}$ on the $x-z$ plane $(z>0$ for the bunch head), with $n_{\mathrm{p}}=1 \times 10^{17} \mathrm{~cm}^{-3}, \sigma_{r}=\sigma_{z}=1 \mu \mathrm{m}$, and $Q_{\mathrm{b}}=30 \mathrm{pC}$.

slice energy spread decrease too. Taking these into account, simulations were performed first with various plasma densities while keeping the laser strength of $a_{0}=\sqrt{2}$. Then for the plasma density of $n_{\mathrm{p}}=1.0 \times 10^{17} \mathrm{~cm}^{-3}$, a higher laser strength $\left(a_{0}=2\right)$ was investigated. The resulted slice energy spread distributions along the beam were shown in Fig. 8(a). The slice energy spread at the buncher center $(\xi=0)$ was shown as a function of the plasma density in Fig. 8(b).

From Fig. 8, it was found that the slice energy spread could be reduced by choosing the proper plasma density and be further mitigated to be below $0.1 \%$ with a stronger laser strength. Figure 8(b) also shows the estimated slice energy spread at the bunch center using Eq. (30), indicating a good agreement between the simulations and the estimations. The deviation, especially at lower plasma density, was probably due to the limits of the linear theory when the beam density is much higher than the plasma density [12].

It is worth noting that for all the distributions in Fig. 8(a), it peaked near the bunch center other than the predicted bunch tail where $E_{\mathrm{z}, \mathrm{b}}$ is supposed to be stronger, as shown in Fig. 2. The inconsistency can be explained by Fig. 9, where a nearly uniform transverse distribution of the longitudinal field driven by a beam was observed near the bunch tail. Because of the much higher beam density than the plasma density, plasma electrons were expelled by the space charge field from the bunch head, leaving a bubble-like channel for the bunch tail and therefore a transversely uniform wakefield, as found in the nonlinear regime [11].

\section{CONCLUSION}

Detailed studies of the effects on the energy spread and slice energy spread of the plasma wakefield driven by laser or particle beam drivers on one side, and of the beam loading effect on the other side allow one to identify the main parameters involved. Although established in the linear regime, the expressions found turn out to be meaningful for reflecting faithfully enough the behavior of the 
accelerated beam as observed in simulations in the quasilinear regime. They have served as very valuable guidance for efficiently minimizing the energy spread and the slice energy spread, leading to beam qualities suitable for driving $\mathrm{x}$-ray free electron lasers. In the context of the EuPRAXIA project, the energy spread was minimized by varying the bunch length at injection and the slice energy spread was optimized by tuning the plasma density and the laser strength. The results from 3D Particle-In-Cell simulations were compared with the theory and a final energy spread less than $1 \%$ and slice energy spread less than $0.1 \%$ were obtained.

\section{ACKNOWLEDGMENTS}

This work has received funding from the European Union's Horizon 2020 research and innovation program under Grant Agreement No. 653782. For numerical simulations, the authors were granted access to the High Performance Computing resources of [Très Grand Centre de Calcul du CEA/Centre Informatique National de l'Enseignement Supérieur/Institut du Développement et des Ressources en Informatique Scientifique] under the allocation A3 made by Genci Grand Équipement National de Calcul Intensif.

\section{APPENDIX: DISTRIBUTION OF THE AMPLITUDE OF THE BETATRON OSCILLATION}

For a Gaussian beam, the distributions of the beam size and divergence are

$$
\begin{aligned}
& f_{X}(x)=\frac{1}{\sqrt{2 \pi} \sigma_{x}} e^{-x^{2} / 2 \sigma_{x}^{2}}=\frac{1}{\sqrt{2 \pi \beta \varepsilon_{\mathrm{rms}}}} e^{-x^{2} / 2 \beta \varepsilon_{\mathrm{rms}}}, \\
& f_{X^{\prime}}\left(x^{\prime}\right)=\frac{1}{\sqrt{2 \pi} \sigma_{x^{\prime}}} e^{-x^{\prime 2} / 2 \sigma_{x^{\prime}}^{2}}=\sqrt{\frac{\beta}{2 \pi \varepsilon_{\mathrm{rms}}}} e^{-\beta x^{\prime 2} / 2 \varepsilon_{\mathrm{rms}}},
\end{aligned}
$$

respectively, where for the Twiss parameters the relation $\beta \gamma=1$ has been used for a matched beam.

The amplitude of the betatron oscillation in $\left(x, x^{\prime}\right)$ trace space is given by $A_{x}=\sqrt{x^{2}+\beta^{2} x^{\prime 2}}$. To get its distribution, we use the following statistical laws [25]: (1) Let $X$ be a continuous random variable with a PDF $f_{X}(x)$ defined over $-\infty<x<\infty$. And, let $Y=u(X)$ be a continuous, monotonic function of $X$ with inverse function $X=v(Y)$. Then, the PDF of $Y$ is given by

$$
f_{Y}(y)=\left|v^{\prime}(y)\right| f_{X}(v(y)) .
$$

If $u(x)$ is a piecewise monotonic function, then $f_{Y}(y)=$ $\sum_{j=1}^{n}\left|v_{j}^{\prime}(y)\right| f_{X}\left(v_{j}(y)\right)$, where $v_{j}(y)$ is the $j$ th subfunction. (2) Let $X$ and $Y$ be two independent continuous random variables with $\operatorname{PDF} f_{X}(x)$ and $f_{Y}(y)$ defined over $(-\infty, \infty)$, respectively. Then, the PDF of $Z=X+Y$ is given by

$$
f_{Z}(z)=\int_{-\infty}^{\infty} f_{X}(x) f_{Y}(z-x) d x
$$

Using Eq. (A3), we can first obtain the PDF of $Z_{1}=x^{2}$ and $Z_{2}=\beta^{2} x^{\prime 2}$. Then the PDF of $Z=Z_{1}+Z_{2}$ is calculated using Eq. (A4). Since $A_{x}=\sqrt{Z}$, its PDF is derived by using Eq. (A3) again and is

$$
f_{A_{x}}\left(A_{x}\right)=\frac{A_{x}}{\sigma_{x}^{2}} e^{-\frac{A_{x}^{2}}{2 \sigma_{x}^{2}}}
$$

In the $\left(y, y^{\prime}\right)$ trace space, we have $A_{y}=\sqrt{y^{2}+\beta^{2} y^{\prime 2}}$ and its p.d.f is

$$
f_{A_{y}}\left(A_{y}\right)=\frac{A_{y}}{\sigma_{y}^{2}} e^{-\frac{A_{y}^{2}}{2 \sigma_{y}^{2}}} .
$$

[1] T. Tajima and J. M. Dawson, Laser Electron Accelerator, Phys. Rev. Lett. 43, 267 (1979).

[2] W. Lu, M. Tzoufras, C. Joshi, F. S. Tsung, W. B. Mori, J. Vieira, R. A. Fonseca, and L. O. Silva, Generating multi$\mathrm{GeV}$ electron bunches using single stage laser wakefield acceleration in a 3D nonlinear regime, Phys. Rev. ST Accel. Beams 10, 061301 (2007).

[3] E. Esarey, C. B. Schroeder, and W. P. Leemans, Physics of laser-driven plasma-based electron accelerators, Rev. Mod. Phys. 81, 1229 (2009).

[4] W. P. Leemans, A. J. Gonsalves, H.-S. Mao, K. Nakamura, C. Benedetti, C. B. Schroeder, C. Tóth, J. Daniels, D. E. Mittelberger, S. S. Bulanov, J.-L. Vay, C. G. R. Geddes, and E. Esarey, Multi-GeV Electron Beams from CapillaryDischarge-Guided Subpetawatt Laser Pulses in the SelfTrapping Regime, Phys. Rev. Lett. 113, 245002 (2014).

[5] Z. Huang, Y. Ding, and C. B. Schroeder, Compact X-ray Free-Electron Laser from a Laser-Plasma Accelerator Using a Transverse-Gradient Undulator, Phys. Rev. Lett. 109, 204801 (2012).

[6] C. B. Schroeder, E. Esarey, C. G. R. Geddes, C. Benedetti, and W. P. Leemans, Physics considerations for laserplasma linear colliders, Phys. Rev. ST Accel. Beams 13, 101301 (2010).

[7] R. Assmann and J. Grebenyuk, in Proceedings of IPAC2014, Dresden, Germany, 2014 (JACoW, Geneva, Switzerland, 2014), pp. 961-964.

[8] P. A. Walker, P. Alesini, A. Alexandrova, M. P. Anania, N. Andreev, I. Andriyash, A. Aschikhin, R. Assmann, T. Audet, A. Bacci et al., Horizon 2020 EuPRAXIA design study, J. Phys. Conf. Ser. 874 (2017).

[9] T. Katsouleas, S. Wilks, P. Chen, J. Dawson, and J. Su, Part. Accel. 22, 81 (1987).

[10] M. Tzoufras, W. Lu, F. S. Tsung, C. Huang, W. B. Mori, T. Katsouleas, J. Vieira, R. A. Fonseca, and L. O. Silva, Beam 
Loading in the Nonlinear Regime of Plasma-Based Acceleration, Phys. Rev. Lett. 101, 145002 (2008).

[11] M. Tzoufras, W. Lu, F. Tsung, C. Huang, W. Mori, T. Katsouleas, J. Vieira, R. Fonseca, and L. Silva, Beam loading by electrons in nonlinear plasma wakes, Phys. Plasmas 16, 056705 (2009).

[12] W. Lu, C. Huang, M. Zhou, W. Mori, and T. Katsouleas, Limits of linear plasma wakefield theory for electron or positron beams, Phys. Plasmas 12, 063101 (2005).

[13] X. Li, A. Mosnier, and P. A. P. Nghiem, Nucl. Instrum. Methods Phys. Res., Sect. A (2018).

[14] M. Reiser, Theory and Design of Charged Particle Beams (John Wiley \& Sons, New York, 2008).

[15] P. Tomassini, S. De Nicola, L. Labate, P. Londrillo, R. Fedele, D. Terzani, and L. A. Gizzi, The resonant multi-pulse ionization injection, Phys. Plasmas 24, 103120 (2017).

[16] P. Lee, G. Maynard, T. Audet, B. Cros, R. Lehe, and J.-L. Vay, Optimization of laser-plasma injector via beam loading effects using ionization-induced injection, Phys. Rev. Accel. Beams 21, 052802 (2018).

[17] J. Vieira, S. Martins, V. Pathak, R. Fonseca, W. Mori, and L. Silva, Magnetic Control of Particle Injection in Plasma Based Accelerators, Phys. Rev. Lett. 106, 225001 (2011).
[18] J. Vieira, J. Martins, V. Pathak, R. Fonseca, W. Mori, and L. Silva, Magnetically assisted self-injection and radiation generation for plasma-based acceleration, Plasma Phys. Controlled Fusion 54, 124044 (2012).

[19] T. Silva et al., "Downramp injection" (to be published).

[20] A. Giribono, A. Bacci, E. Chiadroni, A. Cianchi, M. Croia, M. Ferrario, A. Marocchino, V. Petrillo, R. Pompili, S. Romeo et al., Nucl. Instrum. Methods Phys. Res., Sect. (2018).

[21] C. Benedetti, C. Schroeder, E. Esarey, and W. Leemans, Quasi-matched propagation of ultra-short, intense laser pulses in plasma channels, Phys. Plasmas 19, 053101 (2012).

[22] K. Floettmann, Adiabatic matching section for plasma accelerated beams, Phys. Rev. ST Accel. Beams 17, 054402 (2014).

[23] I. Dornmair, K. Floettmann, and A. R. Maier, Emittance conservation by tailored focusing profiles in a plasma accelerator, Phys. Rev. ST Accel. Beams 18, 041302 (2015).

[24] J.-L. Vay, C. G. R. Geddes, E. Esarey, C. B. Schroeder, W. P. Leemans, E. Cormier-Michel, and D. P. Grote, Modeling of $10 \mathrm{GeV}-1 \mathrm{TeV}$ laser-plasma accelerators using Lorentz boosted simulations, Phys. Plasmas 18, 123103 (2011).

[25] Distributions of functions of random variables, https:// onlinecourses.science.psu.edu/stat414/node/127/. 